\title{
Evaluation of Adiponectinin Patients with Type 2 Diabetes Mellitus in Erbil City
}

\author{
Ashraf N. Kakoo ${ }^{1}$, Nabeel E. Waheda ${ }^{2}$ \\ ${ }^{1}$ Biology Department, College of Science, Salahaddin University, Iraq \\ ${ }^{2}$ Microbiology Department, College of Medicine, Hawler Medical University, Iraq
}

\begin{abstract}
The current study focused to assess Adiponectin in patients afflicted with type 2 diabetes mellitus, it was carried out in the Martyr Layla Qasm Centre for diabetes in Erbil city during the period from April to July 2012.A total of 100 blood samples were collected from patients with age range between (30-79) years and Fifty persons as control with the same age range. Serum Adiponectin was measured by ELISA method. Measurements of Random Blood Sugar (RBS) and Glycated Hemoglobin (HbA1c) also have been performed. This study revealed a highly significant $(p=0.000)$ decrease in mean concentration of adiponectin in patients as compared to control group.
\end{abstract}

Keywords: Diabetic patients, Adiponectin, RBS \& HbA1c

\section{Introduction}

Diabetes mellitus (DM) is a metabolic disorder resulting from a defect in insulin secretion, insulin action, or both, insulin deficiency in turn leads to chronic hyperglycaemia with disturbances of carbohydrate, fat and protein metabolism. (Bastaki, 2005). There are two main types of DM, type 1 insulin dependent diabetes mellitus (IDDM), which is usually manifested in childhood or adolescence, and characterized by pancreatic $\beta$-cell destruction mediated by immune mechanisms, severe insulinopenia and dependence on exogenous insulin to preserve life, whereas type 2 non insulin dependent diabetes mellitus (NIDDM), is characterized by insulin resistance, with association and often progressive defect in insulin secretion, it occurs after age 40 , but it may occur at any age, other types of diabetes could be gestational, or secondary due to other diseases (Saleh, 2011). Adiponectin is a 244 amino acid collagen like protein that is solely secreted by adipocytes and acts as a hormone with anti inflammatory and insulin sensitizing properties (Li et al., 2009). Structurally, Adiponectin has a carboxyl-terminal globular domain and an amino-terminal collagen domain and is structurally similar to complement $1 \mathrm{q}$ which belongs to a family of proteins (Kadowakiet al., 2006). Adiponectin concentration is reversely associated with type $2 \mathrm{DM}$, coronary artery disease and obesity, all together called the metabolic syndrome. Adiponectin decreases blood glucose and free fatty acid serum concentrations and increases insulin sensitivity (Duntaset al., 2004). The production of adiponectin by adipocytes is inhibited by pro-inflammatory factors, such as TNF- $\alpha$ and IL-6. (Aprahamian and Sam, 2011). Evidence suggests that adiponectin has anti-atherogenic properties by improving endothelial function and having anti-inflammatory effects in the vascular wall. In addition, adiponectin modifies vascular intracellular redox signalling and exerts indirect antioxidant effects on human myocardium.(Antoniadeset al., 2009).

\section{Materials and Methods}

\subsection{Study Design}

One hundred of diabetic patients who were admitted to the Martyr LaylaQasmcentre in Erbil city evaluated in this study during the period from April to July 2012Out of 100 patients $65(65 \%)$ were female while $45(45 \%)$ represent male, their age ranged between (30-79) years. Fifty healthy individual (30 female \&20 male), with same age range served as a control group.

\subsection{Blood Sampling}

Blood sampling was performed by withdrawing $5 \mathrm{ml}$ venous blood from each patient and control group using disposable syringe (venipuncture technique). $2.5 \mathrm{ml}$ of the blood were collected in heparinized tube (EDTA) for the performed Hemoglobin A1c (HbA1c) test and remaining blood were collected in vaccination tube with gel, allowed to clot at room temperature then centrifuged at 2500 round per minute for five minute, the sera obtained were divided into two parts, the first part was analyzed for random glucose, The second part of sera kept in a freezer $\left(-20 \mathrm{C}^{\circ}\right)$ to perform serum adiponectin test.

\subsection{Measurement of serum glucose}

Using enzymatic and colorimetric method GOD/POD. This enzymatic test used for quantitative determination of glucose in human or plasma according to plasmatic glucose kit.

\subsection{Quantitative colorimetric determination of} glycohemoglobin (HbA1c) in whole blood.

\subsection{Quantitative determination of serum Adiponectin}

Estimation of Adiponectin was done by Enzyme-Linked ImmunoSorbent Assay (ELISA) technique, which obtained from Merocodia Company (Sweden) 


\section{International Journal of Science and Research (IJSR) \\ ISSN (Online): 2319-7064 \\ Index Copernicus Value (2013): 6.14 | Impact Factor (2015): 6.391}

\subsection{Statistical Analysis}

SPSS (Statistical Package for Social Science) version 16 was used for data entry and analysis. Data are expressed as mean \pm S.E., independent student t-test was used to compare between means of two different samples. The attained values of $\mathrm{P}$ value $<0.05$ were considered statistically significant.

\section{Results}

3.1 Mean concentration of random blood glucose (mg/dl) in sera of study groups.

There was highly significant difference $(p=0.000)$ in the mean concentration of patients and control group, 258.81 \pm 8.1 and $93.05 \pm 1.8$ respectively. (Table 1 )

Table 1: Mean concentration of random blood glucose $(\mathrm{mg} / \mathrm{dl})$ in study groups

\begin{tabular}{|l|l|c|c|}
\hline Study groups & No. & Mean \pm SE & Statistical evaluation \\
\hline Patients & 100 & $258.81 \pm 8.1$ & \multirow{2}{*}{$\mathrm{P}=0.000$} \\
\hline Control & 50 & $93.05 \pm 1.8$ & \\
\hline
\end{tabular}

\subsection{Mean \% of HbA1c category in study groups.}

The number of patients having poor control (HbA1c> 7.6) were higher, when compared with the other category of HbA1c ( Table 2)

Table 2: Mean \% of HbA1c category in study groups

\begin{tabular}{|c|c|c|}
\hline Category of HbA1c & No. & Mean \\
\hline Good control (5.5-6.8) & 8 & 6.181 \\
\hline Fair control (6.9-7.6) & 17 & 7.335 \\
\hline Poor control > 7.6 & 75 & 10.11 \\
\hline Control & 50 & 5.905 \\
\hline
\end{tabular}

\subsection{Estimation of Adiponectin ( $\mathrm{ng} / \mathrm{ml})$ in sera of study group}

The mean concentration of Adiponectin in sera of patients represented low concentration $0.608 \pm 0.01$, when compared with control group $7.36 \pm 0.284$ with highly significant elevation ( $\mathrm{p}=0.000)$. (Table 3 )

Table 3: Mean concentration of Adiponectin (ng/ml) in sera of study group.

\begin{tabular}{|c|c|c|c|}
\hline Study groups & No. & Mean \pm SE & Statistical evaluation \\
\hline Patients & 100 & $0.60 \pm 0.01$ & \multirow{2}{*}{$\mathrm{P}=0.000$} \\
\hline Control & 50 & $7.36 \pm 0.28$ & \\
\hline
\end{tabular}

\section{Discussion}

\subsection{Mean \% of HbA1c category in study groups}

This study demonstrated that the high number 75 of patients have poor control (HbA1c > 7.6), as compared with other level of HbA1c categories. Similar results have been reported by a study, which reported that three quarter of the type 2 diabetes mellitus patients had poor controlled level of HbA1c (Ismail et al, 2011). Another previous study found that Poor control was detected in the majority of the patients with diabetes (Al-Ghamdi, 2004). A key element in diabetes management is glycemic control, which may be indicated by a person's HbA1c. The hemoglobin A1C test measures the percent of glucose in the bloodstream attached to hemoglobin molecules in the previous two to three months, which is the lifespan of red blood cells. Thus, the more excess glucose in the bloodstream, the higher the percentage of hemoglobin molecules attached, the higher the HbA1c level (Davila, 2010). Longer DM duration, diabetic retinopathy, diabetic nephropathy, cigarette smoking, and uncontrolled glycemic levels were significantly associated with higher levels of HbA1c (Mbenza et al., 2008). Good glycemic control has been associated with fewer diabetic complications and better metabolic control. macrovascular and microvascular complications are related to poor glycemic control (Delamater, 2006).

\subsection{Adiponectin and Type $2 \mathrm{DM}$.}

The result in the present study showed that the mean concentration of adiponectin decrease significantly in patients as compared with control group. The above results are similar to results obtained from many other studies. One of them showed that the mean baseline of adiponectin was lower in the diabetic subjects than in the non diabetic subjects (Snehalatha et al., 2003). Another group of worker found decreased serum levels of adiponectin in the type 2 DM patients (Daimonet al., 2003).

Findings from animal studies and metabolic studies in humans suggest several mechanisms through which adiponectin may decrease the risk of type $2 \mathrm{DM}$, including suppression of hepatic gluconeogenesis, stimulation of fatty acid oxidation in the liver, stimulation of fatty acid oxidation and glucose uptake in skeletal muscle, and stimulation of insulin secretion, these effects may be partly mediated by stimulatory effects of adiponectin on signaling pathways for 5_adenosine monophosphate-activated protein kinase and peroxisome proliferator-activated receptor $\alpha$ ( $\mathrm{Li}$ et al., 2009).

A study recorded that In vitro, adiponectin suppressed adhesion molecule expression on endothelial cells, reduced vascular inflammatory responses by inhibition of endothelial cell nuclear factor (NF-kB) signaling, and suppressed macrophage function. In vivo, adiponectin adhered to endothelial lesions and prevented neointimal formation and atherosclerosis in mice, adiponectin thus seems to have protective metabolic and anti-inflammatory properties (Engeliet al., 2003).

Haluziket al., 2004 reported that administration of the adiponectin was accompanied by a weight loss and a decrease of plasma glucose, free fatty acids and triglycerides in mice consuming a high-fat/high-sucrose diet.

\section{Conclusion}

From the present study, we can conclude that baseline serum adiponectin concentrations were statistically significantly, inversely associated with risk of type 2 diabetes. 


\section{References}

[1] Al- Gamdi A A. (2004). Role of HbA1c in management of diabetes mellitus.Saudi Med J., 25(3): 345-5.

[2] Antoniades C, Antonopoulos A S, Tousoulis D and Stefanadis C. (2009). Adiponectin: from obesity to cardiovascular disease. Obesity Review,10: 269-279.

[3] Aprahamian T R and Sam F. (2011).Adiponectin in cardiovascular inflammation and obesity.Int $J$ Inflam, Doi: $10.4061 / 376909$.

[4] Bastaki S. (2005). Diabetes mellitus and its treatment.Int J Diabetes and Metabolism, 13: 111-134.

[5] Daimon M, Oizumi T, Saitoh A, Kameda W, Hirata A, Yamaguchi, H, Ohnuma, H, Igarashi $\mathrm{M}$, Tominiga $\mathrm{M}$ and Kato T. (2003). Decreased serum levels of adiponectin are a risk factor for the progression to type 2 diabetes in the Japanese population. Diabetes Care, 26: 2015-2020.

[6] Davila E P. (2010). Glycemic control: risk factors, quality of life, workforce participation, and mortality among US adults with type 2 diabetes. M.Sc. thesis.Florida International University, Miami, Florida.

[7] Delamater A. (2006). Clinical use of hemoglobin A1C to improve diabetes management. Clinical Diabetes, 24(1): 6 - 8 .

[8] Duntas L H, Popovic V and Panotopoulos G. (2004).Adiponectin: Novelties in metabolism and hormonal regulation. NutrNeurosci, 7: 195-200.

[9] Haluzik M, Parizkova J and HaluzikM M. ( 2004). Adiponectin and its role in the obesity-induced insulin resistance and related complications.Physiol Res, 53: 123-129.

[10] Ismail H, Hanafiah M, Dlah S, Ms S, Huda T and Yunus M. (2011). Control of glycosylated haemoglobin (HbA1c) among type 2 diabetes mellitus patients attending an urban health clinic in Malaysia. MHSJ; 9: 58-65.

[11] Kadowaki T, Yamauchi T, Kubota N, Hara K, Ueki K and Tobe K. (2006). Adiponectin and adiponectin receptor in insulin resistance, diabetes, and the metabolic syndrome.J. Clin. Invest, 116: 1784-1792.

[12]Li S, Shin H J, Ding E L and Dam R M. ( 2009). Adiponectin levels and risk of type 2 diabetes.JAMA; 302(2):179-188.

[13] Mbenza B L, Muaka M M, Mbenza G, Fuele S M, MbalandaL M, Babkei V N and Sungu J M A. (2008). Risk factors of poor control of HBA1c and diabetic retinopathy: Paradox with insulin therapy and high values of HDL in African diabetic patients. Int $J$ Diabetes \& Metabolism, 16: 69-78.

[14] Saleh K M (2011). Study complement activity and humoral immune response in type 2 diabetes mellitus. Diyala Agriculture Science Journal, 3(1): 34-46.

[15] Snehalatha, C.; Mukesh, B.; Simon, M.; Viswanathan, V.; Haffner, S. M. and Ramachandran, A. (2003). Plasma adiponectin is an independent predictor of type 2 diabetes in Asian Indians. Diabetes Care; 26:32263229. 